\title{
DIFFERENCE IN ACETYLCHOLINE-INDUCED NITRIC OXIDE RELEASE OF ARTERIAL AND VENOUS GRAFTS IN PATIENTS AFTER CORONARY BYPASS OPERATIONS
}

Hiroaki Nishioka, MD

Soichiro Kitamura, MD

Yoichi Kameda, MD

Shigcki Taniguchi, MD

Tetsuji Kawata, MD

Kazumi Mizuguchi, MD

\begin{abstract}
Objectives: In vivo investigation of nitric oxide release in coronary bypass grafts has not been reported. We studied acetylcholine-induced nitric oxide release in vivo of coronary bypass grafts and vasomotor responses to acetylcholine of grafted coronary arteries in patients after coronary bypass grafting. Methods: We examined 24 internal thoracic artery grafts and 16 saphenous vein grafts in 39 patients. The mean ages of the patients were 65 years for the arterial grafts and 68 years for the venous grafts. Nitric oxide was measured as the plasma nitrite level by the Griess reaction. Before and after intragraft acetylcholine infusion $(5 \mu \mathrm{g})$, blood was sampled from the distal end of the graft, and angiograms were taken and analyzed by cine-densitometry. Results: The plasma nitrite concentration after stimulation with acetylcholine compared with the control value was $134 \% \pm 52 \%$ at 4 minutes $(p=0.05)$ and $184 \% \pm 107 \%$ at 6 minutes $(p=0.01)$ in the arterial grafts; in the venous grafts these values were $101 \% \pm 24 \%$ at 4 minutes $(p=0.96)$ and $108 \% \pm 36 \%$ at 6 minutes $(p=0.69)$. Low-dose acetylcholine dilated the coronary arteries supplied by arterial grafts by $6.3 \% \pm 16.6 \%$ whereas coronary arteries supplied by venous grafts were reduced by $9.8 \% \pm 11.8 \%$ in diameter and the vasoactive responses were different $(p=0.01)$. Conclusions: In vivo internal thoracic artery grafts had more endothelium-derived nitric oxide release in response to acetylcholine than did saphenous vein grafts after coronary bypass grafting. (J Thorac Cardiovasc Surg 1998;116:454-9)
\end{abstract}

A terial grafts, especially internal thoracic artery (ITA) grafts, recently have been used more often instead of saphenous vein grafts (SVGs) as the first choice for coronary artery revascularization. ${ }^{1}$ There are many reports that present the advantages of ITA grafts over venous grafts. First, ITA grafts have excellent patency and superior resistance to atherosclerotic changes over the long-term postoperative period. ${ }^{2}$ Second, they adapt physiologically to the flow demands of coronary circulation ${ }^{3}$ and adapt anatomically to physical development in pediatric patients. ${ }^{4}$ These advantages are derived mainly from the viability of the ITA grafts. ${ }^{5}$ In organ bath

From the Department of Surgery III, Nara Medical University, Nara, Japan.

Received for publication June 24, 1997; revisions requested Sept. 24, 1997; revisions received March 9, 1998; accepted for publication March 9, 1998.

Address for reprints: Hiroaki Nishioka, MD, Department of Surgery III, Nara Medical University, 840 Shijo-cho, Kashihara, Nara 634, Japan.

Copyright 1998 by Mosby, Inc.

$0022-5223 / 98 \$ 5.00+0 \quad \mathbf{1 2 / 1 / 9 0 1 8 6}$ experiments, endothelial function in freshly harvested ITAs and saphenous veins in the grafted or ungrafted state differed with respect to the release of nitric oxide (NO). ${ }^{6-8}$ In vivo investigation of $\mathrm{NO}$ release in implanted coronary bypass grafts, however, has not yet been reported. The purpose of this study was to compare the acetylcholine-induced endothelial NO relcase in bypass grafts between ITAs and saphenous veins in patients after coronary artery bypass grafting and to evaluate the metabolic function of arterial and venous grafts.

\section{Patients and methods}

Patients. In 39 patients (36 men and 3 women), 40 bypass grafts were studied during postoperative cardiac catheterization. Patients with any signs of ischemia, angina, heart failure, or stenosis of the bypass grafts were excluded from the study protocol. Patient profiles are shown in Table I. The mean age and mean postoperative period for the patients at the time of this study were $65 \pm$ 9 years (range 47 to 78 years) and $38 \pm 46$ months (range 1 to 154 months), respectively, for the ITA graft group and $68 \pm 4$ years (range 60 to 75 years) and $48 \pm 50$ months (range 1 to 152 months), respectively, for the SVG group. 
Table I. Patient profiles

\begin{tabular}{lccc}
\hline & $I T A$ & SVG & p Value* \\
\hline No. of patients & 23 & 16 & \\
No. of grafts studied & 24 & 16 & \\
Age (yr) & $65 \pm 9$ & $68 \pm 4$ & 0.53 \\
Female & $1(4.2 \%)$ & $2(12.5 \%)$ & 0.55 \\
Coronary risk factors & & & \\
$\quad$ Hypertension & $11(46 \%)$ & $10(63 \%)$ & 0.73 \\
$\quad$ Diabetes mellitus & $8(33 \%)$ & $7(44 \%)$ & 0.66 \\
Hyperlipidemia & $11(46 \%)$ & $8(50 \%)$ & 0.99 \\
$\quad$ History of smoking & $15(63 \%)$ & $13(81 \%)$ & 0.26 \\
Postoperative period (mo) & $38 \pm 46$ & $48 \pm 50$ & 0.45 \\
$\quad$ Range (mo) & $1-154$ & $1-152$ & \\
Distal anastomosis & & & \\
$\quad$ LAD & $15(63 \%)$ & $2(13 \%)$ & \\
LCX & $8(33 \%)$ & $7(44 \%)$ & 0.001 \\
RCA & $1(4 \%)$ & $7(44 \%)$ & \\
\hline
\end{tabular}

$L A D$, left anterior decending; $R C A$, right coronary artery. *SVG vs ITA

There were no significant differences between the two groups in age, gender, postoperative period, or coronary risk factors including hypertension, diabetes mellitus, hyperlipidemia, and smoking history. All of the grafts were used as isolated grafts and all ITAs (19 left ITAs and 5 right ITAs) were used as in situ grafts. For the grafts bypassed to the left circumflex coronary artery (LCX), there were also no significant differences between the two groups in clinical profiles including age, postoperative period, and coronary risk factors. All grafts were angiographically patent with good run-off flow.

Study protocol. The study protocol was approved by the Institutional Review Committee on Human Research of Nara Medical University. Informed consent was obtained from the patients before the procedure and all the studies were completed without complications. Patients underwent cardiac catheterization while in a fasting state without premedication, after interruption of all vasoactive drugs for more than 24 hours. No patients received $\beta$-adrenergic receptor blockers within 48 hours before the study. After postoperative diagnostic catheterization, a $5 \mathrm{~F}$ Judkins catheter for the right coronary artery was positioned at the ostium of the coronary bypass graft, and grafts were selectively cannulated to the distal anastomotic site with a $2.7 \mathrm{~F}$ microcatheter (Tracker-18 infusion catheter, Target Therapeutics) through a guiding catheter. Blood in the distal portion of the graft was obtained through the microcatheter as a control. Blood was aspirated through the microcatheter over about 60 to 90 seconds to avoid hemolysis. Five micrograms or $3 \times 10^{-8}$ mol of acetylcholine solution was then infused into the graft through the guiding catheter for 30 seconds. Blood samples from the distal portion of the graft were obtained in the same manner as for the control, at 4 and 6 minutes after the administration of acetylcholine. Eight minutes after the beginning of acetylcholine administration, angiography of the grafts and the bypassed coronary arteries was performed. Angiograms were not taken in the first five patients in the SVG group or in the first six in the ITA group, but in the remaining patients angiographic inves-
Table II. Plasma nitrite and nitrate levels

\begin{tabular}{lcc}
\hline & ITA $(n=24)$ & $S V G(n=16)$ \\
\hline Plasma nitrite level & & \\
Control $\left(10^{-7} \mathrm{~mol} / \mathrm{L}\right)$ & $8.85 \pm 4.23$ & $9.18 \pm 6.60$ \\
Ratio of $4 \mathrm{~min}$ after ACH & $1.34 \pm 0.52^{*} \dagger$ & $1.01 \pm 0.24$ \\
Ratio of 6 min after ACH & $1.84 \pm 1.07^{*} \neq$ & $1.08 \pm 0.36$ \\
Total plasma nitrite and & & \\
$\quad$ nitrate level & & \\
Control $\left(10^{-7} \mathrm{~mol} / \mathrm{L}\right)$ & $335.0 \pm 179.7$ & $353.1 \pm 281.0$ \\
Ratio of 4 min after ACH & $1.16 \pm 0.51^{*}$ & $0.93 \pm 0.09$ \\
Ratio of 6 min after ACH & $1.08 \pm 0.19^{\text {ss }}$ & $0.93 \pm 0.10$
\end{tabular}

Data presented are mean plus or minus the standard deviation. $A C H$, Acetylcholine.

${ }^{*} p<0.05$ vs SVGs.

${ }^{\mathrm{sis}} p<0.01$ vs SVGs.

${ }^{\dagger} p<0.05$ vs control.

${ }^{*} p<0.01$.

tigations were consecutively performed and the diameters of grafts and coronary arteries at points about $1 \mathrm{~cm}$ upstream and downstream from the anastomotic site were measured by cine-densitometry. The electrocardiogram and arterial pressure were monitored continuously during the procedures. Finally, $1 \mathrm{mg}$ isosorbide dinitrate was injected into the graft through the guiding catheter to overcome the acetylcholine-induced vasoconstriction.

Assay of NO concentrations. Blood samples were obtained through the catheters with the use of disposable syringes and centrifuged as soon as possible at $3000 \mathrm{rpm}$ for 5 minutes at $4^{\circ} \mathrm{C}$. Plasma was stored at $-80^{\circ} \mathrm{C}$ until use. Plasma samples were diluted 1:2 with deionized water and filtered through a $0.45 \mu \mathrm{m}$ microfilter. Plasma nitrite $\left(\mathrm{NO}_{2}{ }^{-}\right)$and nitrate $\left(\mathrm{NO}_{3}{ }^{-}\right)$concentrations were measured with an NOx analyzer (Eno10, Eicom, Kyoto, Japan) with high-performance liquid chromatography and the Griess reaction. ${ }^{9,10}$ In brief, the samples were first passed through a column to separate $\mathrm{NO}_{3}{ }^{-}$from $\mathrm{NO}_{2}{ }^{-}$. Then the samples were passed through a second column containing copper-coated cadmium to reduce the $\mathrm{NO}_{3}^{-}$to $\mathrm{NO}_{2}^{-}$. Next the $\mathrm{NO}_{2}^{-}$was detected by reaction with the Griess reagent $(0.03 \mathrm{~mol} / \mathrm{L}$ sulfanilamide plus $0.15 \mathrm{~mol} / \mathrm{L} \mathrm{HCl}$ solution containing $1.0 \times 10^{-3} \mathrm{~mol} / \mathrm{L} \mathrm{N}-[1$-naphthyl] ethylenediamine). Absorption was measured at $546 \mathrm{~nm}$ with use of a spectrophotometer. Thus the separate $\mathrm{NO}_{2}^{-}$and $\mathrm{NO}_{3}^{--}$levels could be measured simultaneously. The detection limit of the assay was $1.0 \times 10^{-8} \mathrm{~mol} / \mathrm{L}$.

Quantitation of graft and coronary artery diameters. The diameters of the grafts and grafted coronary arteries were calculated by cine-densitometry with a microcomputer analyzing program, Elk Cine-Angio System Cam1000 , produced by Nishimoto Sangyo, Osaka, Japan. ${ }^{11}$

Statistical analysis. Measurements are expressed as mean plus or minus standard deviations (percentiles). Statistical analysis was performed with the SPSS version 6.1.3J program (SPSS, Chicago, Ill.) on a personal computer (XL-590/Compaq Computer). The patient profile data were analyzed by the $\chi^{2}$ or Mann-Whitney $U$ test, and the plasma $\mathrm{NO}_{2}^{-}$and $\mathrm{NO}_{3}{ }^{-}$concentration data were analyzed by the Wilcoxon matched-pairs signed-rank test, Mann-Whitney U test, and analysis of variance (Pillai's 


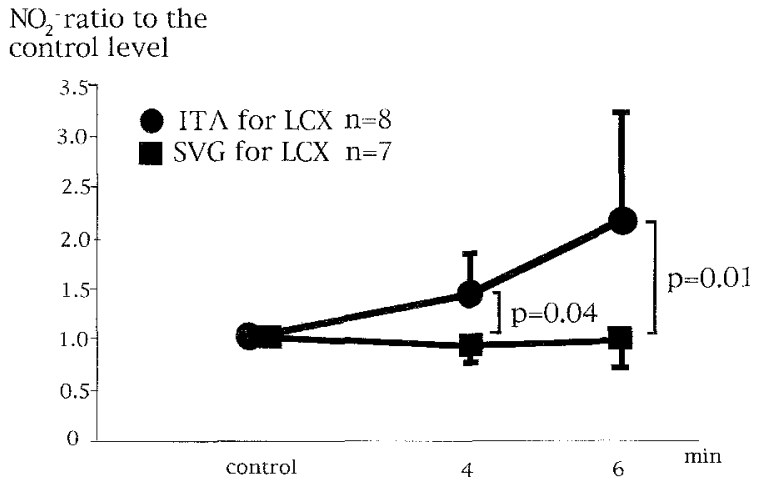

Fig. 1. Plasma $\mathrm{NO}_{2}{ }^{-}$concentration after stimulation with acetylcholine compared with control level for grafts bypassed to the LCX. For grafts to the LCX, plasma $\mathrm{NO}_{2}{ }^{-}$concentration after stimulation with acetylcholine compared with the control value was significantly higher in ITA grafts than in SVGs at 4 minutes $(p=0.04)$ and at 6 minutes $(p=0.01)$. This response was similar to the result for all study grafts, including grafts to other coronary arteries.

multivariate test). Correlation coefficients were calculated by the method of Pearson. All $p$ values are two tailed.

\section{Results}

Plasma $\mathrm{NO}_{2}^{-}$and $\mathrm{NO}_{3}^{-}$concentrations. For 40 grafts in 39 patients who had undergone coronary artery bypass grafting, the plasma $\mathrm{NO}_{2}{ }^{-}$and $\mathrm{NO}_{3}{ }^{-}$ concentrations in the distal portion of the graft after acetylcholine administration are shown in Table II. Plasma $\mathrm{NO}_{2}^{-}$concentration significantly increased after acetylcholine infusion in the ITA grafts (at 4 minutes, $p=0.05$; at 6 minutes, $p=0.01$ ), whereas there were no significant increases for the SVGs (at 4 minutes, $p=0.96$; at 6 minutes, $p=0.69$ ). The response in plasma $\mathrm{NO}_{2}^{-}$levels after stimulation with acetylcholine was different between ITA grafts and SVGs (at 4 minutes, $p=0.04 ; 6$ minutes, $p=$ 0.03 ). The plasma concentration of total $\mathrm{NO}_{2}^{-}$plus $\mathrm{NO}_{3}{ }^{-}$also responded differently to the stimulation of acetylcholine between the two groups (at 4 minutes, $p=0.03 ; 6$ minutes, $p=0.01$ ). Significance of the difference in plasma $\mathrm{NO}_{2}^{-}$concentrations between the ITA grafts and SVGs was statistically borderline by analysis of variance $(p=0.05)$. A significant relation existed in the ratio of plasma level of $\mathrm{NO}_{2}{ }^{-}$to total $\mathrm{NO}_{2}{ }^{-}$plus $\mathrm{NO}_{3}{ }^{-} 6$ minutes after stimulation with acetylcholine $(p=0.05)$.

Because the target coronary arteries of the ITA or SVG differed, and the ITA was mainly used for the left anterior descending coronary artery and the SVG was mainly used for the right coronary artery

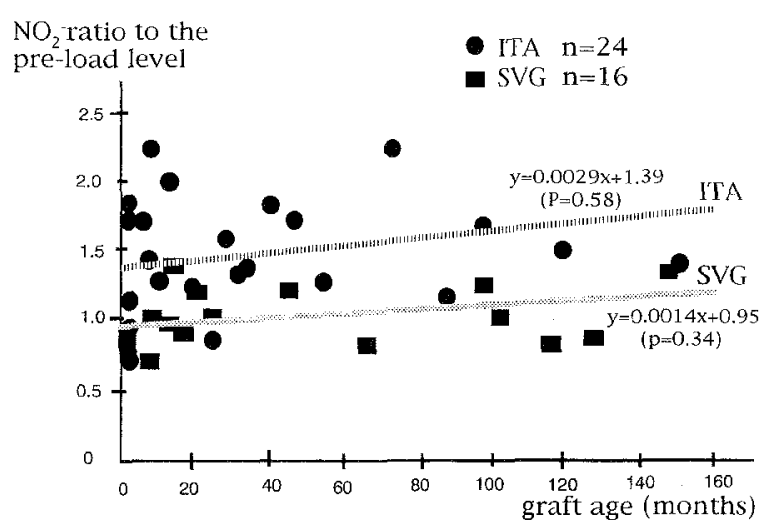

Fig. 2. The relation between the change in nitrite level after acetylcholine infusion and the graft age (period between graft implantation and the study). There was no significant correlation by regression analysis between the change of plasma $\mathrm{NO}_{2}^{-}$level after acetylcholine infusion and the graft age.

and $\mathrm{LCX}$ as shown in Table $\mathrm{I}, \mathrm{NO}_{2}{ }^{-}$responses were compared in the bypass grafts for the LCX where both the ITA and SVG were used. The plasma $\mathrm{NO}_{2}^{-}$ concentration after stimulation with acetylcholine compared with the control value was different between the ITA and SVG (at 4 minutes, $p=0.04$; at 6 minutes, $p=0.01$ ) (Fig. 1). The increase in plasma $\mathrm{NO}_{2}{ }^{-}$levels after stimulation with acetylcholine in the ITAs did not reach statistical significance.

Time between the graft implantation and the study ranged from 1 to 154 months for the ITA group and from 1 to 152 months for the SVG group. However, NO release induced by acetylcholine administration did not differ with regard to the graft age. There were no significant correlations or significant differences by regression analysis between the graft age and ratio of plasma $\mathrm{NO}_{2}{ }^{-}$after stimulation with acetylcholine (Fig. 2).

Vasomotor response to acetylcholine. Fig. 3 shows angiograms of an SVG implanted to the LCX in a 65-year-old man 115 months after bypass grafting in the control condition and after intragraft infusion of $5 \mu \mathrm{g}$ acetylcholine. The native coronary artery was constricted after acetylcholine administration. Fig. 4 shows angiograms of an ITA graft implanted to the left anterior descending artery in a 52-year-old man 145 months after bypass grafting in the control condition and after intragraft infusion of $5 \mu \mathrm{g}$ acetylcholine. The native coronary artery was dilated after acetylcholine administration.

Low-dose acetylcholine dilated ITA grafts by $6.3 \% \pm 11.6 \%$ whereas SVGs were reduced by $3.1 \%$ 

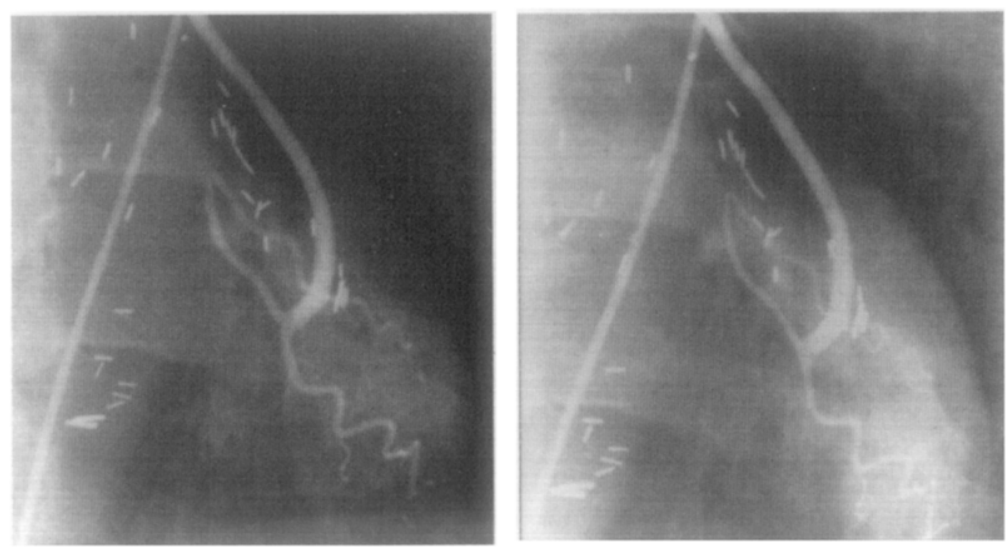

Fig. 3. Angiograms of an SVG implanted to the LCX in a 65-year-old man taken 115 months after the operation in the control condition (left) and after intragraft infusion of $5 \mu \mathrm{g}$ acetylcholine (right). The native coronary artery bypassed with an SVG constricted after acetylcholine administration.
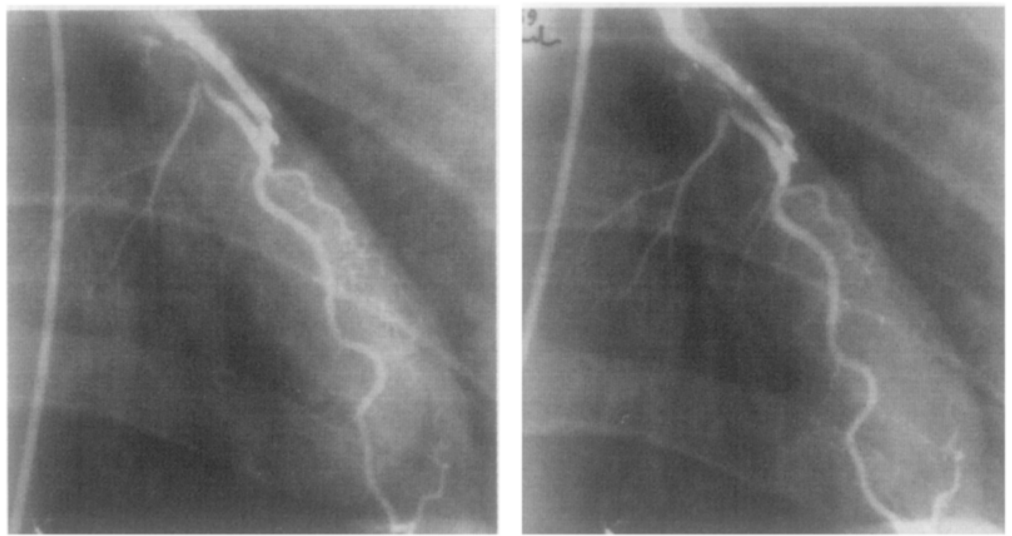

Fig. 4. Angiograms of a left ITA graft implanted to the left anterior descending artery in a 52-year-old man taken 145 months after the operation in the control condition (left) and after intragraft infusion of 5 $\mu \mathrm{g}$ acetylcholine (right). The native coronary artcry bypassed with an ITA graft dilated after acetylcholine administration.

$\pm 8.9 \%$. Vasomotor responses of the grafts after acetylcholine administration were different between the ITA grafts and SVGs $(p=0.02)$ (Fig. 5).

Low-dose acetylcholine dilated the coronary arteries supplied by arterial grafts by $6.3 \% \pm 16.6 \%$ whereas coronary arteries supplied by venous grafts were reduced by $9.8 \% \pm 11.8 \%$ in diameter. Ace tylcholine-induced vasoconstriction was significantly less in the coronary arteries grafted with ITAs than in those with SVGs $(p=0.01)$ (Fig. 6).

\section{Discussion}

Advantages of the ITA graft over the SVG are excellent patency, lower incidence of graft disease in the long-term postoperative period, and physiologic and anatomic adaptability. ${ }^{12-16}$ These advantages are apparently a result of the properties of the vessel, especially of the endothelium. In organ bath experiments, endothelial release of NO was different in ITAs and SVGs in the grafted or ungrafted states. ${ }^{16-18}$ In this study, we demonstrated for the first time a greater release of NO in the ITA graft than in the SVG in patients $41 \pm 47.3$ months after coronary artery bypass grafting.

NO assay. NO cannot be easily or directly measured in biologic tissues or fluids because it is rapidly metabolized. Instead, NO concentration is determined by measurement of $\mathrm{NO}_{2}^{-}$and $\mathrm{NO}_{3}{ }^{-}$concentrations. Determination of total plasma $\mathrm{NO}_{2}^{-}$plus $\mathrm{NO}_{3}{ }^{-}$concentrations as a measurement of $\mathrm{NO}$ con. centration has been previously described and is a well established method. ${ }^{12,13}$ Although $\mathrm{NO}_{2}^{-}$and 


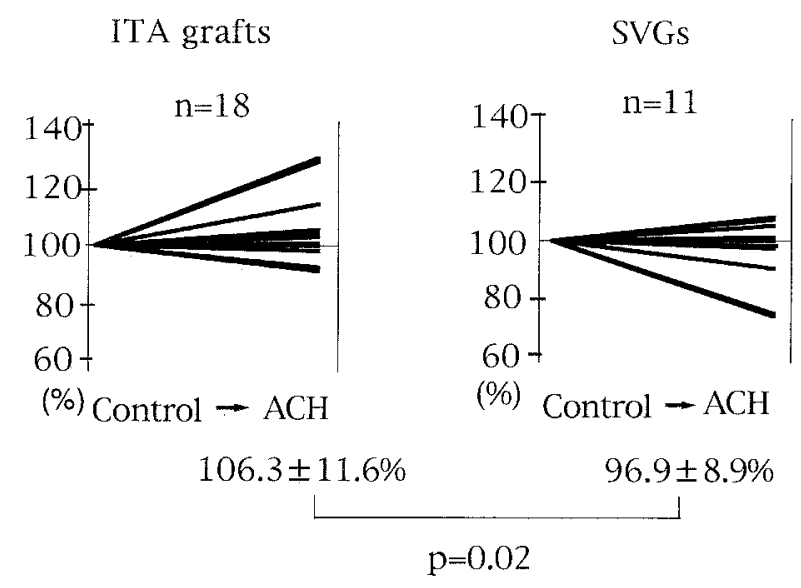

Fig. 5. Vasomotor responses of the grafts to acetylcholine $(A C H)$ infusion. Low-dose acetylcholine dilated ITA grafts whereas the SVGs were reduced in diameter (ITA vs $\mathrm{SVG}, p=0.02$ ).

$\mathrm{NO}_{3}{ }^{-}$are metabolites of $\mathrm{NO}$, the metabolic rate of conversion of $\mathrm{NO}$ to $\mathrm{NO}_{2}{ }^{-}$or $\mathrm{NO}_{3}{ }^{-}$in the blood is not presently known. Plasma $\mathrm{NO}_{2}^{-}$concentration is very low and previous assays rarely succeeded in the measurement of isolated plasma $\mathrm{NO}_{2}^{-}$level as a measurement of $\mathrm{NO}$ production. ${ }^{14}$ Our measurements of separate plasma $\mathrm{NO}_{2}{ }^{-}$and $\mathrm{NO}_{3}{ }^{-}$concentrations were made with instrumentation incorporating high-performance liquid chromatography and the Griess reaction, which enabled separate evaluation of changes in plasma $\mathrm{NO}_{2}^{-}$and $\mathrm{NO}_{3}^{-}$concentrations. ${ }^{9}, 10$

NO is released bidirectionally by the endothelium. ${ }^{15}$ Extraluminal NO acts on the underlying vascular smooth muscle ${ }^{16}$ and inhibits smooth muscle cell proliferation. Intraluminal NO is released into the blood. The results of the concentration measurements of metabolites in the present study demonstrated that the ITA graft actually released NO into the blood in response to acetylcholine. The NOreleasing response to acetylcholine was also better for the ITA grafts to the LCX as compared with that of the SVG for the same arterial lesions. There seem to be no differences in the ability of the graft to produce NO regardless of which coronary artery is targeted.

NO plays an important role in many physiologic functions. NO inhibits platelet aggregation and promotes platelet disaggregation. ${ }^{17}$ It also inhibits platelet and leukocyte adhesions to the endothelium, ${ }^{18}$ protecting the endothelium from injury induced by superoxide radicals; in addition, intralumi-

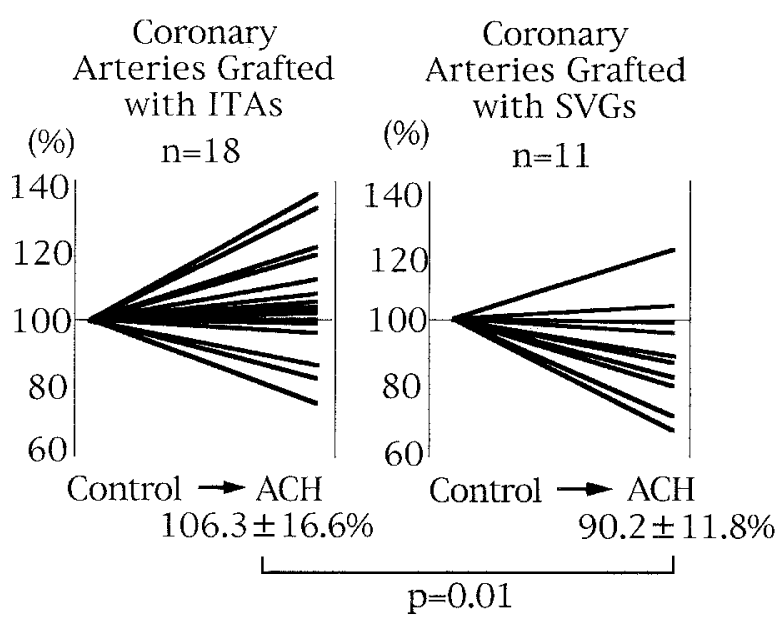

Fig. 6. Vasomotor responses of the grafted coronary arteries to acetylcholine $(A C H)$ infusion. Acetylcholineinduced vasoconstriction was significantly less in the coronary arteries grafted with ITA grafts than in those grafted with SVGs $(p=0.01)$.

nally released NO may have a metabolic effect on the downstream vascular beds. ${ }^{19}$ Therefore ITA grafts with NO-releasing endothelium may have an antiatherosclerotic effect on both grafts and grafted coronary arteries.

Vasomotor response to acetylcholine. ITAs are usually free of atherosclerotic changes ${ }^{20}$ and dilate in response to local administration of acetylcholine as long as the endothelium is functional. ${ }^{21}$ The results of the NO assay offer an explanation for the graft's response to acetylcholine. The endothelial release of $\mathrm{NO}$ in SVGs was insufficient to reverse the direct constrictive effect of acetylcholine. Here we have provided quantitative data comparing the response to intragraft administration of low-dose acetylcholine of coronary arteries grafted with the I'TA and SVG. The results are consistent with those of a previous angiographic study that examined the vasomotor response of grafts and coronary arteries grafted with ITAs to the administration of ergonovine or substance P. ${ }^{22,23}$ However, high-dose acetylcholine administration can uniformly induce a constrictive response of the coronary artery, and a dilative response can be induced by an optimal dose of acetylcholine. ${ }^{24}$ Several reasons for the dilation of the coronary arteries bypassed with ITA grafts in this study can be considered. First, intraluminally released NO from the graft can affect the downstream coronary arteries; second, endothelium-dependent NO-mediated dilation of the coronary ar- 
tery occurs in response to acetylcholine; and third, flow-mediated vasodilation after the increased flow of the graft occurs in response to acetylcholine or the angiographic procedures. Although we cannot exclude other mechanisms of coronary artery dilation such as an involvement of prostacyclin ${ }^{25}$ or endothelium-dependent hyperpolarization factor, ${ }^{26}$ the results of the NO assay in the present study offer an explanation of the coronary artery response by the endothelium-derived NO from the grafts. The present angiographic results may suggest that the ITA graft has a possible metabolic effect of protecting the grafted coronary arteries from spasm and atherosclerosis.

In summary, the ITA graft is not only an excellent conduit with good long-term patency, but it also has a potential metabolic effect that protects the graft and the grafted coronary arteries from spasm and atherosclerosis. This is the first study to demonstrate in human subjects that in situ ITA grafts can produce more NO compared with venous grafts after coronary bypass grafting. Thus coronary artery bypass grafting with the ITA may have the salutary effect of transplanting functional endothelial cells into the coronary circulation.

\section{REFERENCES}

1. Loop FD, Lytle BW, Cosgrove DM, et al. Influence of the internal-mammary-artery graft on 10-year survival and other cardiac events. N Engl J Med 1986;314:1-5.

2. Cameron A, Green GE, Brogno DA, Thornton J. Internal thoracic artery grafts: 20-year clinical follow-up. J Am Coll Cardiol 1995;25:188-92.

3. Morita R, Kitamura S, Kawachi K, et al. Exercise coronary flow reserve of bilateral internal thoracic artery bypass grafts. Ann Thorac Surg 1993;55:883-7.

4. Kitamura S, Seki T, Kawachi K, Morita R, Kawata T. Excellent patency and growth potential of internal mammary artery grafts in pediatric coronary bypass surgery: new evidence for a "live" conduit. Circulation 1989;78(Suppl):I29-39.

5. Werner GS, Wuchwald A, Kreuzer H, Wiegant V. Evidence in vivo of an intact endothelial function in internal mammary arteries before and after implantation as coronary grafts. Coron Artery Dis 1990;1:461-8.

6. Lüscher TF, Diederich D, Siebenmann R, et al. Difference between endothelium-dependent relaxation in arterial and venous coronary grafts. N Engl J Med 1988;319:462-7.

7. Ku DD, Claufield JB, Kirklin JK. Endothelium-dependent response in long-term human coronary artery bypass grafts. Circulation 1991;83:402-11.

8. Ignarro LJ, Buga GM, Wood KS, Byrns RE, Chaudhuri G. Endothelium-derived relaxing factor produced and released from artery and vein is nitric oxide. Proc Natl Acad Sci U S A 1987;84:9265-9.

9. Green LC, Wagner DA, Grogowsk J, Skippers JS, Wishnock JS, Tannenbaum SR. Analysis of nitrate, nitrite and $[15 \mathrm{~N}]$ nitrate in biological fluids. Anal Biochem 1982;126:131-8.
10. Muscara MN, de Nucci G. Simultaneous determination of nitrite and nitrate anion in plasma, urine and cell culture supernatants by high-performance liquid chromatography with post-column reactions. J Chromatogr B Biomed Sci Appl 1996;686:157-64.

11. Seki T, Kitamura S, Kawachi K, et al. A quantitative study of postoperative luminal narrowing of the internal thoracic artery graft in coronary artery bypass surgery. J Thorac Cardiovasc Surg 1992;104:1532-8.

12. Marletta MA, Yoon PS, Iyengar R, Leaf CD, Wishnock JS. Macrophage oxidation of L-arginine to nitrite and nitrate: nitric oxide is an intermediate. Biochemistry 1988;27:8706-11.

13. Zaballos GA, Bernstein RB, Tompson CI, et al. Pharmacodynamics of plasma nitrate/nitrite as an indication of nitric oxide formation in conscious dog. Circulation 1995;91:2982-8.

14. Roth E, Steininger R, Winkler $S$, et al. I-Arginine deficiency after liver transplantation as an effect of arginase efflux from the graft. Transplantation 1994;57:665-9.

15. Pearson PJ, Evora PRB, Schaff HV. Bioassay of EDRF from internal mammary arteries: implications for early and late bypass graft patency. Ann Thorac Surg 1992;54:1078-84.

16. Moncada S, Palmer RMJ, Higgs EA. Nitric oxide: physiology, pathophysiology and pharmacology. Pharmacol Rev 1991;43: $109-42$.

17. Radomski MW, Palmer RMJ, Moncada S. The anti-aggregating properties of vascular endothelium: interactions between prostacyclin and nitric oxide. Br J Pharmacol 1987;92:639-46.

18. Radomski MW, Palmer RMJ, Moncada S. Endogenous nitric oxide inhibits human platelet adhesion to vascular endothelium. Lancet 1987;2:1057-8.

19. Kalm M, Schrader J. Control of coronary vascular tone by nitric oxide. Circ Res 1990;66:1561-75.

20. Kobayashi H, Kitamura S, Kawachi K, Morita R, Konishi Y, Tsutsumi M. A pathological and biological study of arteriosclerosis in the internal thoracic artery, a vessel commonly used as a graft in coronary artery bypass surgery. Jpn J Surg 1993;23:697-703.

21. Takeda T, Fujita M, Fudo T, et al. Response of internal mammary artery to local administration of acetylcholine. Am J Cardiol 1996;77:302-4.

22. Kitamura S, Morita R, Kanji K, et al. Different response of coronary and internal mammary artery bypass grafts to ergonovine and nitroglycerin in variant angina. Ann Thorac Surg 1989;47:756-60.

23. Kushwaha SS, Bastami M, Tadjkarimi S, Ilsley CDJ, Mitchell $A G$, Yacoub $M H$. Late endothelial function of free and pedicled internal mammary artery grafts. J Thorac Cardiovasc Surg 1995;110:453-62.

24. Hanet C, Robert A, Wijins W. Vasomotor response to ergometrine and nitrates of saphenous vein grafts, internal mammary artery grafts, and grafted coronary arteries late after bypass surgery. Circulation 1992;86(Suppl):II210-6.

25. Forstermann U, Muggy A, Alheid U, Haverich A, Frolich JC. Selective attenuation of endothelium-mediated vasodilation in atherosclerotic human coronary artery. Circ Res 1988;62:18590.

26. Horzmann S, Kikovetz WR, Windischhofer W, Paschke E, Graier WF. Pharmacologic differentiation between endothelium dependent relaxations sensitive and resistant to nitro- $\mathrm{L}$ arginine in coronary arteries. J Cardiovasc Pharmacol 1994; 23:747-56. 